\title{
Calidad del aire en galpón avícola con ventilación natural durante la fase de pollitos
}

\author{
Robinson Osorio H. ${ }^{1}$, Ilda F. F. Tinoco ${ }^{1}$, Jairo A. Osorio S. ${ }^{2}$, \\ Cecília de F. Souza ${ }^{1}$, Diogo J. de R. Coelho ${ }^{1} \&$ Fernanda C. de Sousa ${ }^{1}$ \\ ${ }^{1}$ Universidade Federal de Viçosa/Departamento de Engenharia Agrícola/Construções Rurais e Ambiência. Viçosa, MG. E-mail: robinson.hernandez@ufv.br / \\ rosorio0@unal.edu.co (Corresponding author); iftinoco@ufv.br; cfsouza@ufv.br; diogo.coelho@ufv.br; fernanda.sousa@ufv.br \\ ${ }^{2}$ Universidad Nacional de Colombia/Departamento de Ingeniería Agrícola y Alimentos/Construcciones Rurales. Medellín, Antioquia. E-mail: aosorio@unal.edu.co
}

\section{Palabras clave: estrés por frio bioclimatología aislamiento térmico manejo de cortinas}

\begin{abstract}
R E S U M E N
Los pollos de engorde durante la primera fase deben estar protegidos del estrés por frio, especialmente en el inverno, noches y madrugadas frías. Para proteger a los animales, los avicultores han utilizado diferentes estrategias, entre ellas está el uso de varias capas de cortinas para aumentar el nivel de aislamiento térmico y disminuir las pérdidas de calor, disminuyendo también la permeabilidad con el aire externo. En este contexto, es importante dar especial atención a la calidad del aire interno de los galpones avícolas como parte de una producción bajo parámetros de bienestar animal. El presente estudio fue realizado en el municipio de Barbacena, MG, en Brasil, y tuvo como objetivo evaluar la calidad del aire al nivel de los pollos y los trabajadores, específicamente de las concentraciones de amoniaco, dióxido de carbono y monóxido de carbono, en un galpón avícola naturalmente ventilado durante la primera fase de vida de pollos de engorde en el período de inverno, y la influencia del nivel de aislamiento con cortinas laterales sobre las concentraciones de amoniaco. Durante la primera semana experimental, los niveles de amoniaco sobrepasaron los valores aceptables, y se encontró diferencia estadística entre las concentraciones de amoniaco para los diferentes niveles de aislamiento con cortinas laterales.
\end{abstract}

Key words:

cold stress

bioclimatology

thermal insulation

curtains management

\section{Air quality in a poultry house with natural ventilation during phase chicks}

\begin{abstract}
A B S T R A C T
Broilers during the first phase should be protected from cold stress, especially in the winter, cold nights and early mornings. To protect animals, poultry producers have used different strategies, including the use of multilayer of curtain, to increase the thermal insulation and reduce heat losses, also decreasing the permeability to the external air. In this context it is important to give special attention to the indoor air quality of poultry sheds as part of a production under animal welfare parameters. This study was conducted in the municipality of Barbacena, MG, in Brazil, and aimed to assess air quality at the level of the chickens and workers, specifically concentrations of ammonia, carbon dioxide and carbon monoxide, in a poultry house naturally ventilated during the first phase of life of broilers in the winter period, and the influence of insulation level with side curtains on concentrations of ammonia. During the first experimental week, ammonia levels exceed acceptable values, and statistical difference was found between the concentrations of ammonia for different insulation levels with side curtains.
\end{abstract}




\section{INTRODUCCIÓN}

Por ser un país de clima predominantemente cálido y con poca amplitud térmica, la avicultura industrial de Brasil (y de América Latina), opto por instalaciones con poco o ningún aislamiento térmico en paredes laterales, utilizando ventilación natural, y posibilitando en otros casos el uso de sistemas híbridos de ventilación mecánica (Nazareno et al., 2009; Menegali et al., 2013; Mendes et al., 2014; ). Se habla de galpones sin aislamiento térmico ya que la resistencia térmica de las cortinas laterales de polipropileno es aproximadamente $0.045 \mathrm{~m}^{2}{ }^{\circ} \mathrm{kW}^{-1}$, según IVE (2011), se considera material no aislante térmico cuando su resistencia térmica es menor o igual a $0.25 \mathrm{~m}^{2} \mathrm{~kW}^{-1}$.

Este tipo de instalaciones han sido económicas y eficientes para resolver problemas de estrés por calor en pollos adultos, pero si se considera que durante la fase de pollitos, estos deben estar protegidos de estrés por frio y corrientes de aire intensas, en el inverno, noches y madrugadas frías (Cordeiro et al., 2010; Campos et al., 2013), hay dudas si la falta de aislamiento térmico compromete el ambiente interno (Osorio et al., 2013). En este periodo es necesario evitar la pérdida de calor hacia el ambiente externo, generalmente en detrimento de la renovación y calidad del aire interno (Vigoderis et al., 2010; Damasceno et al., 2014).

Como resultado del metabolismo animal, del proceso de descomposición de la cama y del uso de calefactores de combustión, se genera calor, humedad, gases nocivos, y polvo (Osorio et al., 2012; Baracho et al., 2013). El deterioro de la calidad del aire puede afectar la salud y el bienestar de los animales y los trabajadores y constituye un riesgo (Curtis, 1983; Wathes,1999; OWH, 2005; Nääs et al., 2007; Vigoderis et al., 2010).

En este contexto es importante dar especial atención a la calidad del aire en el interior de los galpones avícolas como parte de la producción bajo parámetros de bienestar animal (Vigoderis et al., 2010; Robins \& Phillips, 2011)., también bajo normas internacionales sobre calidad y emisión de gases de efecto invernadero (Tinôco et al., 2004; Osorio et al., 2009; Vigoderis et al., 2010).

Estudios recientes han demostrado poca eficiencia del control de calidad de aire en galpones avícolas en la fase inicial de producción, y que se requieren realizar mayores evaluaciones en tipologías típicas de aviarios de países tropicales y subtropicales con el fin de realizar propuestas de mejoramiento de estas instalaciones (Cordeiro et al., 2010; Campos et al., 2013). Así, el objetivo de este trabajo fue evaluar la calidad del aire al nivel de los pollos y de los trabajadores, en términos de las concentraciones de amoniaco $\left(\mathrm{NH}_{3}\right)$, dióxido de carbono $\left(\mathrm{CO}_{2}\right)$ y monóxido de carbono $(\mathrm{CO})$, en un galpón avícola naturalmente ventilado durante la primera fase de vida de las aves durante el período de inverno y la influencia del nivel de aislamiento con cortinas sobre las concentraciones de $\mathrm{NH}_{3}$.

\section{Material y Métodos}

Este estudio se desarrolló en un galpón avícola con una población de 40.000 aves, orientado este - oeste, localizado en el municipio de Barbacena-MG, en Brasil (latitud 21 $1^{\circ} 6^{\prime} 18^{\prime \prime}$ S, longitud $43^{\circ} 46^{\prime} 12^{\prime \prime} \mathrm{W}$, altitud $1.126 \mathrm{~m}$ ). El clima de la región es clasificado como templado suave (Cwa) según Köppen. Las dimensiones de la instalación son: $18 \mathrm{~m}$ de ancho, por $120 \mathrm{~m}$ de largo, altura del cielo raso $2.45 \mathrm{~m}$, y cama de cascarilla de café de quinto uso. El experimento se desarrolló durante el mes de julio (periodo de invierno en el hemisferio sur) con temperatura media de $16{ }^{\circ} \mathrm{C}$, máxima de $32{ }^{\circ} \mathrm{C}$ y mínima de $4{ }^{\circ} \mathrm{C}$.

La densidad de aves fue aproximadamente de 55 aves $\mathrm{m}^{-2}$ durante la primera semana, 36 aves $\mathrm{m}^{-2}$ para la segunda y de 18 aves $\mathrm{m}^{-2}$ a partir de la tercera semana. El sistema de calefacción estaba compuesto por cuatro calefactores Debona-90, con una potencia de $95040 \mathrm{kcal}$ o $110.5 \mathrm{~kW}$ cada una, que permanecieron encendidos todo el tiempo durante la primera fase. El galpón fue dotado de tres capas de cortinas para disminuir las pérdidas de calor del galpón al exterior una

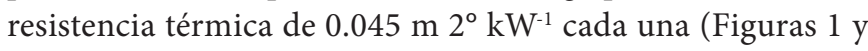
2). El sistema de ventilación mecánica no fue accionado, por lo que durante el experimento funciono con ventilación natural.

Fueron colectados los datos de concentración de $\mathrm{CO}, \mathrm{CO}_{2}$ y $\mathrm{NH}_{3}$ en la entrada de aire y en el punto central al interior del galpón, a la altura de $0.20 \mathrm{~m}$ (referente para los pollitos) y 1.50 $\mathrm{m}$ (referente para el trabajador), de forma semi-continua cada $3 \mathrm{~h}$, es decir, en 8 horarios, $24 \mathrm{~h}$ por día, durante julio de 2011, anotando los tiempos de abertura y cerramiento de cortinas.

Los datos de concentración de $\mathrm{NH}_{3}$ se colectaron con un sensor digital BW GasAlert $\mathrm{NH}_{3}$ Extrems BW-GAXT-A-DL, que tiene una faja de 0 a $100 \mathrm{ppm}$. Las concentraciones de $\mathrm{CO}_{2}$ fueron medidas con un sensor AZ 77535, con faja de medición de: 0 a 9999 ppm. Los niveles de CO se midieron con un sensor Carbon Monoxide Meter Model, modelo 7701, con faja de medición de 0 a 999 ppm.

Los datos de temperatura se colectaron gracias a una red de sensores (de $10.5 \times 4.0 \mathrm{~m}$ de distancia entre sensor y sensor) conectados entre sí por medio del sistema $1-$ Wire $^{\mathrm{TM}}$. Los sensores se conectaron a un computador, y se realizó la adquisición de datos por el software STRADA desarrollado por Rocha et al. (2008).

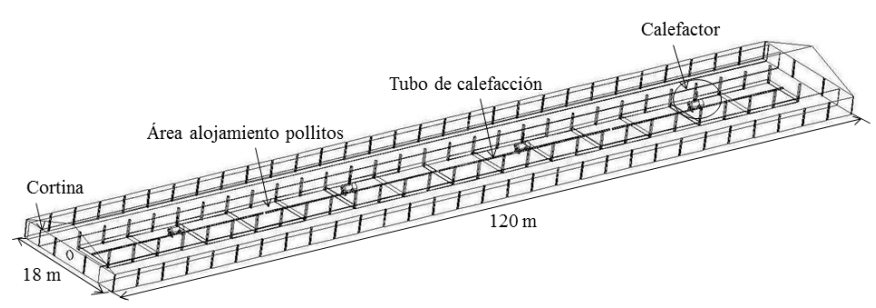

Figura 1. Esquema del galpón experimental

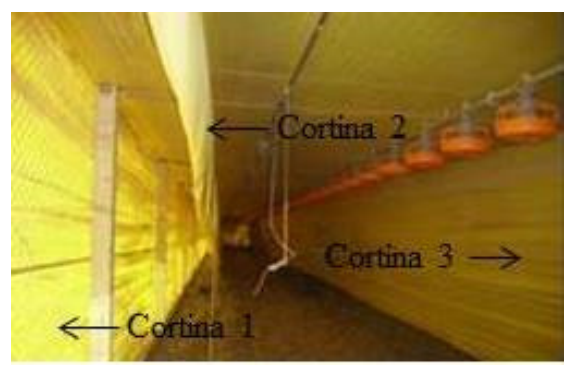

Figura 2. Disposición de cortinas dentro del galpón para aislamiento térmico 
Para observar el efecto del aislamiento térmico de las capas de cortinas, se supuso un material compuesto como si las capas de cortinas de polietileno y aire fuesen placas tipo sándwich, para esto fue utilizado el método de simplificación de capas de materiales (INMETRO, 2013), para el cálculo del espesor y la resistencia térmica equivalente.

Fue realizado un análisis grafico de la temperatura interna y externa horaria, además de análisis de datos medios tomados cada $3 \mathrm{~h}$ para cada una de las tres semanas experimentales de la concentración de gases de $\mathrm{NH}_{3}, \mathrm{CO}$ y $\mathrm{CO}_{2}$, y un análisis grafico para las concentraciones de $\mathrm{NH}_{3}$ y $\mathrm{CO}_{2}$ a cada $3 \mathrm{~h}$ durante la primera semana experimental. También se realizó un análisis estadístico por medio análisis de variancia enteramente casualizado y de test de medias con ayuda del programa " $R$ " para observar la influencia del número de cortinas que aislaron térmicamente el galpón sobre su calidad del aire al nivel de los animales y del trabajador, siendo este un galpón naturalmente ventilado. La Tabla 1 muestra los diferentes tratamientos utilizados.

Tabla 1. Tratamientos estadísticos y descripción

\begin{tabular}{ccc}
\hline Tratamientos & Numero de capas de cortinas & Nivel $(\mathbf{m})$ \\
\hline T1 & 3 & 0.20 \\
T2 & 3 & 1.50 \\
T3 & 2 & 0.20 \\
T4 & 2 & 1.50 \\
T5 & 1 & 0.20 \\
T6 & 1 & 1.50 \\
\hline
\end{tabular}

\section{RESUlTAdos y Discusión}

En la Figura 3 se observa el comportamiento de la temperatura media dentro y fuera del galpón para las tres primeras semanas de vida de las aves, además de las líneas que delimitan la faja de confort para las mismas según Curtis (1983) y Abreu \& Abreu (2011). Se observa que durante toda la primera y segunda semana, aunque el sistema de calefacción estuvo accionado permanentemente, en ningún momento la temperatura media del galpón estuvo dentro de la zona de temperaturas de confort, lo que según Curtis (1983) y Abreu \& Abreu (2011) puede aumentar el consumo de alimento, disminuir conversión alimenticia y generar riesgo de enfermedades respiratorias. Durante la tercera semana solo se alcanzaron temperaturas medias adecuadas en las horas

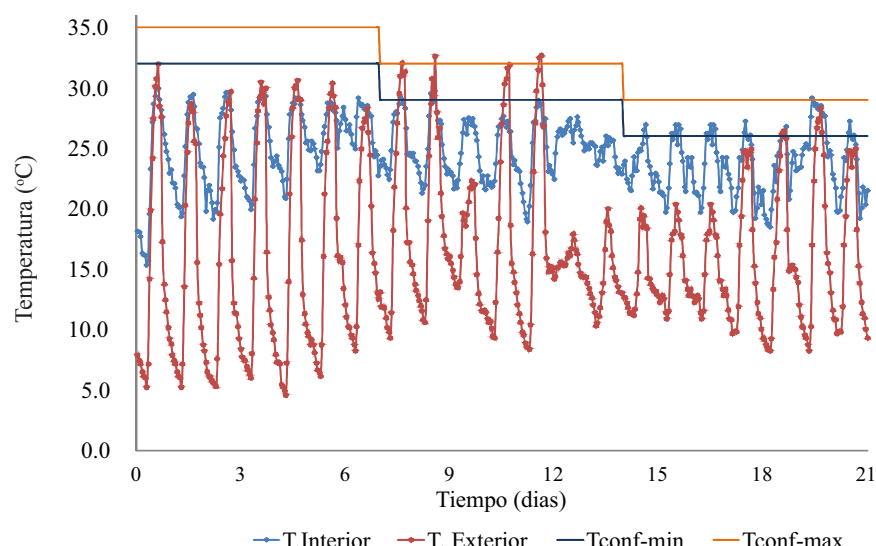

Figura 3. Variación de la temperatura media dentro y fuera del galpón durante la primera semana experimental más cálidas del día, esto posiblemente porque el sistema de calefacción estaba sub-dimensionado, lo que llevó a que no fuera accionado el sistema de ventilación mínima durante la primera fase.

El uso de cortinas simples o una sola cortina en las paredes laterales de los galpones avícolas puede comprometer el confort térmico de las aves en la primera fase en épocas o periodos fríos (Osorio et al., 2013), por lo que muchas veces son utilizadas varias cortinas para aumentar el aislamiento térmico. En la Tabla 2 se observa el efecto de las capas de cortinas sobre el aislamiento térmico, donde con dos capas se logra superar el valor de $0.25 \mathrm{~m}^{2} \mathrm{~kW}^{-1}$ (IVE, 2011) logrando aislar térmicamente el galpón. Esto explica el efecto aislante, y por qué esta técnica es comúnmente practicada por productores de Brasil y América Latina.

La Tabla 3 muestra los datos de valores medios de concentraciones de gases durante las tres primeras semanas de vida de las aves. En esta tabla se observan valores de concentración de $\mathrm{NH}_{3}$ particularmente altos durante la primera semana experimental, sobrepasando el valor máximo de 25 ppm para aves (Wathes et al., 1998) y de humanos (HPA, 2007), mientras que los valores de CO estuvieron cercanos a cero y las concentraciones medias de $\mathrm{CO}_{2}$ no alcanzaron el límite máximo de 3000 ppm (Wathes, 1999). Los altos niveles de $\mathrm{NH}_{3}$ y los valores de $\mathrm{CO}_{2}$ superiores a 2000 ppm durante la primera semana, pueden ser perjudiciales (Wathes, 1999) y son producto de la combinación de la reutilización de la cama (de quinto uso), unido al hecho de brindar mayor aislamiento del ambiente exterior con el uso de varias capas de cortinas que reducen la permeabilidad del aire interno hacia el externo, concordando con planteamientos de Vigoderis et al. (2010).

Aunque se pretende en Brasil y América Latina, en la medida de lo posible que los galpones avícolas trabajen con ventilación natural todo el tiempo para ahorrar energía eléctrica e inversión en equipos, en condiciones de frio, y bajo la condición de mayor aislamiento por causa de varias capas de cortinas (lo que trae consigo menor permeabilidad del galpón con el aire externo), la ventilación natural con ventilación mecánica, para alcanzar tazas

Tabla 2. Resistencia térmica para diferentes capas de cortinas de polietileno

\begin{tabular}{|c|c|c|c|c|}
\hline \multicolumn{2}{|c|}{ Capas } & \multicolumn{3}{|c|}{ Resistencia térmica $\left(\mathrm{m}^{2}{ }^{\circ} \mathrm{kW}^{-1}\right)$} \\
\hline Cortinas & Aire & $\begin{array}{c}\text { Cortina } \\
\text { polietileno }\end{array}$ & $\begin{array}{c}\text { Cámara de aire } \\
\text { no ventilada espesor }>5 \mathrm{~cm}\end{array}$ & Total \\
\hline 1 & 0 & 0.045 & 0.17 & 0.045 \\
\hline 2 & 1 & 0.045 & 0.17 & 0.260 \\
\hline 3 & 2 & 0.045 & 0.17 & 0.475 \\
\hline
\end{tabular}

Tabla 3. Datos de concentraciones medias de gases en ppm

\begin{tabular}{ccccc}
\hline \multirow{2}{*}{ Semana } & Gas & Entrada & \multicolumn{2}{c}{ Galpón (m) } \\
\cline { 4 - 5 } 1 & de aire & $\mathbf{0 . 2 0}$ & $\mathbf{1 . 5 0}$ \\
\cline { 4 - 5 } 1 & $\mathrm{NH}_{3}$ & $0.3+/-1.0$ & $42.3+/-12.2$ & $44.1+/-11.97$ \\
& $\mathrm{CO}_{2}$ & $723.4+/-45.4$ & $2015.9+/-624.7$ & $2191.6+/-582.0$ \\
& $\mathrm{CO}_{2}$ & $0.0+/-0.0$ & $2.1+/-5.0$ & $3.7+/-7.1$ \\
& $\mathrm{NH}_{3}$ & $0.0+/-0.0$ & $12.6875+/-5.1$ & $12.5+/-4.3$ \\
2 & $\mathrm{CO}_{2}$ & $736.1+/-43.1$ & $2026.8+/-673.3$ & $2126.1+/-704.0$ \\
& $\mathrm{CO}_{2}$ & $0.1+/-0.5$ & $1.0+/-2.2$ & $2.0+/-4.1$ \\
& $\mathrm{NH}_{3}$ & $0.0+/-0.0$ & $14.4+/-4.3$ & $14.9+/-5.3$ \\
3 & $\mathrm{CO}_{2}$ & $711.6+/-54.7$ & $2547.1+/-856.7$ & $2756.9+/-871.2$ \\
& $\mathrm{CO}$ & $0.0+/-0.0$ & $0.3+/-1.0$ & $0.8+/-3.0$ \\
\hline
\end{tabular}


de ventilación mínima que garanticen los niveles adecuados de calidad del aire para las aves (Nazareno et al., 2009; Menegali et al., 2013; Mendes et al., 2014).

En la Figura 4 se observa el comportamiento en el tiempo de la concentración de $\mathrm{NH}_{3}$ para la primera semana experimental. La ventilación mínima ayuda a reducir las concentraciones de gases en el interior de los galpones (Vigoderis et al., 2010), por lo que alrededor de las $15 \mathrm{~h}$ se presentaron los valores mínimos de concentración tanto a la altura del pollo como a la altura del trabajador, debido al uso de un solo par de cortinas y a su apertura $(0.50 \mathrm{~m})$ para renovar el aire, lo que permitió higienizar parte del aire del galpón.

Los horarios con mayor concentración de $\mathrm{NH}_{3}$ fueron de 6:00 a 9:00 h y de 18:00 a 21:00 h (Figura 4), esto se debe a que en estos horarios el galpón se encontraba aislado térmicamente con tres capas de cortinas, en la mañana el ambiente empieza a calentarse y la cama era de cascara de café de quinto uso lo que favorece mayor liberación de $\mathrm{NH}_{3}$ de la cama hacia la atmosfera interna (Zapata et al., 2015), llegando a valores de $55 \mathrm{ppm}$ a los $0.20 \mathrm{~m}$ (altura del animal) y $60 \mathrm{ppm}$ a los 1.50 $\mathrm{m}$ (altura referente para los trabajadores). En el inicio de la noche el galpón aun permanecía cálido producto de su inercia térmica, lo que también favoreció la mayor liberación de $\mathrm{NH}_{3}$ llegando a niveles de 59 ppm a los $0.20 \mathrm{~m}$ y 63 ppm a los $1.50 \mathrm{~m}$, valores altamente peligrosos tanto para los animales (Wathes et al., 1998; Oliveira \& Monteiro, 2013) como para los trabajadores (HPA, 2007).

En la Tabla 4 se muestra el análisis estadístico de la influencia del número de cortinas que aíslan térmicamente el aviario sobre los niveles de concentración media de $\mathrm{NH}_{3}$ al interior del galpón. Se observa que no existe diferencia estadística en concentración en los niveles de 0.20 y $1.50 \mathrm{~m}$ en ninguna de las tres condiciones, es decir, no existe diferencia estadística entre T1 y T2, T3 y T4, T5 y T6. Sin embargo, se aprecia que a mayor número de cortinas que aíslan el galpón, aumentan los contenidos de amoniaco, mostrando diferencia

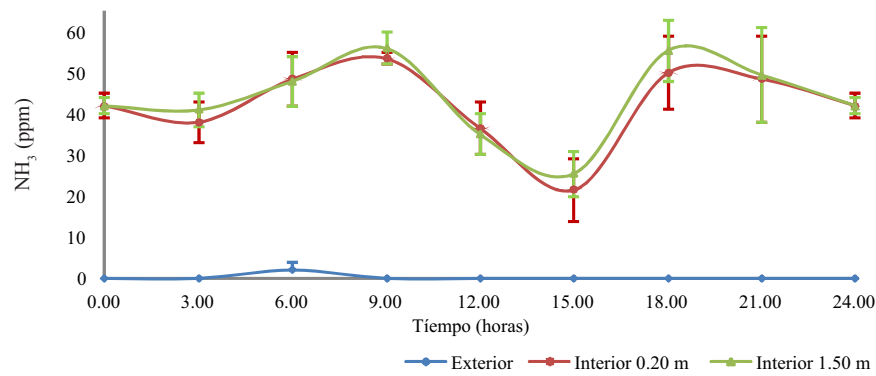

Figura 4. Valores médios de concentracion de $\mathrm{NH}_{3}$ Primera semana experimental

Tabla 4. Datos de concentraciones medias de $\mathrm{NH}_{3}$ en ppm

\begin{tabular}{crrccr}
\hline Tratamientos $^{*}$ & N & Media & Mínimo & Máximo & $\begin{array}{c}\text { Desv. } \\
\text { est. }\end{array}$ \\
T1 & 11 & $47.3 \mathrm{a}$ & 33.0 & 59.0 & 9.1 \\
T2 & 11 & $48.7 \mathrm{a}$ & 37.0 & 63.0 & 9.6 \\
T3 & 4 & $31.5 \mathrm{~b}$ & 19.0 & 43.0 & 12.3 \\
T4 & 4 & $30.7 \mathrm{~b}$ & 15.0 & 48.0 & 15.8 \\
T5 & 17 & $13.7 \mathrm{C}$ & 3.0 & 30.0 & 7.0 \\
T6 & 17 & $14.5 \mathrm{C}$ & 4.0 & 31.0 & 7.3 \\
\hline
\end{tabular}

Las medias unidas por la misma letra no difieren entre sí, al 0,05 de probabilidad por el test de Tukey $(\mathrm{P} \leq 0.001, \mathrm{~F}=39.06)$

* Ver Tabla 1 estadística entre los contenidos medios de $\mathrm{NH}_{3}$ con el uso de un (T5 y T6), dos (T3 y T4) y tres pares de cortinas (T1 y T2). Solo con el uso de un par de cortinas se apreció niveles de concentración adecuados para los animales (Wathes et al., 1998; Oliveira \& Monteiro, 2013) y trabajadores (HPA, 2007), inferiores a 25 ppm.

Este comportamiento se debe por una parte a que la cama del aviario era de quinto uso, pero principalmente a la menor permeabilidad con el aire externo producto del mayor aislamiento del exterior y a la no utilización de ventilación mecánica para realizar ventilación higiénica o mínima, ya que en este caso se optó por reducir el estrés por frio de los animales a costa de la calidad del aire interna.

En la Figura 5 se puede observar que el comportamiento de la concentración $\mathrm{CO}_{2}$ en el interior del galpón fue similar al mostrado por el $\mathrm{NH}_{3}$, canzando valores mínimos de concentración en las horas del mediodía, cuando el galpón tuvo mayor permeabilidad con el ambiente externo, y alcanzando el valor máximo promedio de 2429 ppm a 0.20 m y 2543 ppm a $1.50 \mathrm{~m}$ a las 9:00 h, con valor máximo absoluto de 3114 ppm a $0.20 \mathrm{~m}$ y $3037 \mathrm{ppm}$ a $1.50 \mathrm{~m}$, por lo tanto tornandose un ambiente peligroso en este horario para los animales y trabajadores según Wathes (1999), Jones et al. (2005) e HPA (2007). Los mismos autores recomiendan tener cuidado en ambientes con niveles por encima de 2000 ppm, lo que sugiere que en este galpón, solo se presentó un ambiente adecuado para animales y trabajadores, en las horas cercanas al medio dia. Esto comfirma el efecto del numero de capas de cortinas sobre la permeabilidad y concentración de gases al interior del galpón, y por lo tanto sobre su calidad del aire.

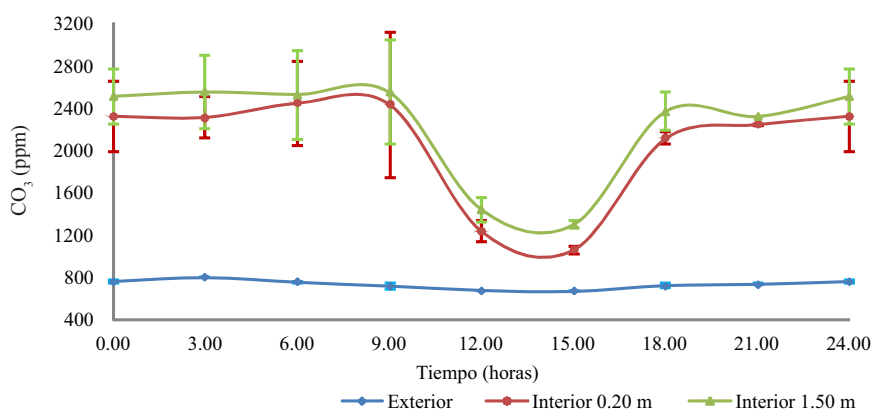

Figura 5. Valores médios de concentracion de dióxido de carbono $\left(\mathrm{CO}_{2}\right)$ durante la primera semana experimental

\section{Conclusiones}

1. No se encontró diferencia significativa en las concentraciones de amoniaco a nivel del animal y del trabajador para la misma condición de aislamiento con cortinas; sin embargo se encontró que a mayor nivel de aislamiento con cortinas, la concentración de amoniaco aumento, mostrando diferencia significativa en las concentraciones para un, dos y tres pares de cortinas.

2. Durante la primera semana de vida de los pollitos, los niveles de $\mathrm{NH}_{3}$ estuvieron por encima de los límites adecuados tanto para los animales como para los trabajadores debido a la no utilización de ventilación mínima o higiénica por medios mecánicos y a la reutilización de la cama.

3. Los niveles de $\mathrm{CO}$ estuvieron próximos a cero dentro del galpón producto de una adecuada combustión de 
los calefactores al interior del galpón. Los niveles de $\mathrm{CO}_{2}$ presentaron un comportamiento similar al presentado por el $\mathrm{NH}_{3}$, presentando los mayores niveles en horas de la noche y la mañana, cuando el galpón es encontraba con mayor nivel de aislamiento (mayor número de cortinas).

\section{Agradecimientos}

Los autores agradecen al Departamento de Engenharia Agrícola de la Universidade Federal de Viçosa, CAPES, FAPEMIG, CNPq, al Departamento de Ingeniería Agrícola y Alimentos de la Universidad Nacional de Colombia Sede Medellín, y a Nogueira Rivelli ${ }^{\circledast}$ por su invaluable ayuda en el desarrollo de esta investigación.

\section{Literatura Citada}

ABNT, 15220-2. Desempenho Térmico de Edificações-Parte 2: Métodos de cálculo da transmitância térmica, da capacidade térmica, do atraso térmico e do fator de calor solar de elementos e componentes de edificações. 2003. <http://www.labeee.ufsc.br/sites/default/ files/projetos/normalizacao/Termica_parte2_SET2004.pdf $>3$ Mar. 2015.

Abreu, V. M. N.; Abreu, P. G. Os desafios da ambiência sobre os sistemas de aves no Brasil. Revista Brasileira de Zootecnia, v.40, p.1-14. 2011.

Baracho, M. S.; Cassiano; J. A.; Nääs, I. A. Tonon, G. S.; Garcia, R. G.; Royer, A. F. B.; Santana, M. R. Ambiente interno em galpões de frango de corte com cama nova e reutilizada. Agrarian, v.6, p.473-478. 2013.

Campos, A. T.; Klosowski, É. S.; Sousa, F. A.; Ponciano, P. F.; Navarini, F. C.; Yamagi Junior, T. Eficiência de sistema de aquecimento auxiliar para aviários, com base nos índices de conforto térmico. Bioscience Journal, v.29, p.703-711. 2013.

Cordeiro, M. B.; Tinôco, I. de F.; Silva, J. N.; Vigoderis, R. B.; Pinto, F. A. C.; Cecon, P. R. Conforto térmico e desempenho de pintos de corte submetidos a diferentes sistemas de aquecimento no período de inverno. Revista Brasileira de Zootecnia, v.39, p.217-224. 2010. http://dx.doi.org/10.1590/S1516-35982010000100029

Curtis, S. E. Environmental management in animal agriculture. 1.ed. Ames: Iowa State University Press. 1983. 409p.

Damasceno, F. A.; Osorio Saraz, J. A.; Mendes, L. B.; Martin, S.; Martins, M. A. Evaluation of a heating system in poultry houses using a CFD model. Revista Facultad Nacional de Agronomia, v.67, p.7355-7363, 2014. http://dx.doi.org/10.15446/rfnam.v67n2.44178

HPA - Health Protection Agency (Ammonia - Toxicological overview. 2007. <https://www.gov.uk/government/uploads/system/uploads/ attachment_data/file/337506/hpa_ammonia__Toxicological_ Overview_v2.pdf>. 10 Mar. 2015.

INMETRO, I. N. de M. Qualidade e tecnologia. Anexo V - Portaria n.o 50, de 01 de fevereiro de 2013. 2013. <http://www.inmetro. gov.br/consumidor/produtosPBE/regulamentos/AnexoV.pdf>. 15 Jan. 2015.

IVE - Instituto Valenciano de la Edificación. Catálogo de Soluciones Constructivas de Rehabilitación Energética. 2011. <https://www. five.es>. 1 Mar. 2015.

Jones, E. K.; Wathes, C. M.; Webster, A. J. F. Avoidance of atmospheric ammonia by domestic fowl and the effect of early experience. Applied Animal Behaviour Science, v.90, p.293-308. 2005. http:// dx.doi.org/10.1016/j.applanim.2004.08.009
Mendes, L. B.; Tinôco, I. de F. F.; Ogink, N. W.; Rocha, K. S.; Osorio, S. J. A.; Santos, M. S. Ammonia emissions from a naturally and a mechanically ventilated broiler house in Brazil. Revista Brasileira de Engenharia Agrícola e Ambiental, v.18, p.1179-1185. 2014. http://dx.doi.org/10.1590/1807-1929/agriambi.v18n11p1179-1185

Menegali, I.; Tinôco, I. de F. F.; Carvalho, C.; Souza, C. F.; Martins, J. H. Comportamento de variáveis climáticas em sistemas de ventilação mínima para produção de pintos de corte. Revista Brasileira de Engenharia Agrícola e Ambiental, v.17, p.106-113. 2013. http:// dx.doi.org/10.1590/S1415-43662013000100015

Nääs, I. A.; Miragliotta, M. Y.; Baracho, M. S.; Moura, D. Ambiência aérea em alojamento de frangos de corte: Poeira e gases. Engenharia Agrícola, v.27, p.326-335. 2007. http://dx.doi.org/10.1590/S010069162007000300001

Nazareno, A. C.; Pandorfi, H.; Almeida, G. L.; Giongo, P. R.; Pedrosa, E. M.; Guiselini, C. Avaliação do conforto térmico e desempenho de frangos de corte sob regime de criação diferenciado. Revista Brasileira de Engenharia Agrícola e Ambiental, v.13, p.802-808. 2009. http://dx.doi.org/10.1590/S1415-43662009000600020

Oliveira, P. A. V. de; Monteiro, A. N. T. R. Emissão de amônia na produção de frangos de corte. In: Conferência Facta, Campinas, 2013. Anais...Campinas: Facta, 2013. <http://ainfo.cnptia.embrapa. br/digital/bitstream/item/91032/1/final7197.pdf>. 5 Mar. 2015.

Osorio, H. R.; Tinôco, I. de F. F.; Osorio, J. A.; Rocha, K. S. O.; Garcia, L. M. G. Modelamiento del ambiente térmico y aéreo de un galpón de presión negativa tipo túnel para pollitos. Revista Facultad Nacional de Agronomia, v.66, p.7085-7093, 2013.

Osorio, J. A.; Aredes M. M.; Zapata, O. L.; Damasceno, F.; Ciro, H. J. Una revisión acerca de la dinámica de fluidos computacionales (CFD) en instalaciones avícolas. Dyna, v.79, p.142-149. 2012.

Osorio, J. A.; Tinôco, I. de F. F.; Ciro, H. J. Ammonia: A review of concentration and emission models in livestock structures. Dyna, v.76, p.89-99. 2009.

Robins, A.; Phillips, C. J. C. International approaches to the welfare of meat chickens. World's Poultry Science Journal, v.67, p.351-369. 2011. http://dx.doi.org/10.1017/S0043933911000341

Rocha, K. S. O.; Martins, J. H.; Tinôco, I. de F. F.; Melo, E. C.; Lopes, D. C.; Hermsdorff, W. Remote environmental monitoring and management of data systems. Central Theme, Technology for All: Sharing the Knowledge for Development. In: International Conference of Agricultural Engineering, 37, Brazilian Congress of Agricultural Engineering, International Livestock Environment Symposium-ILES, 8, 2008, Iguassu Falls City, Proceedings... Iguassu Falls City: International Commission of Agricultural Engineering (CIGR), Institut fur Landtechnik), 2008. Not paginated

Tinôco, I. de F. F.; Figueiredo, J. L. A.; Santos, R. C.; Silva, J.; Pugliesi, N. L. Placas porosas utilizadas em sistemas de resfriamento evaporativo. Revista Engenharia na Agricultura, v.12, p.17-23. 2004.

Vigoderis, R. B.; Cordeiro, M. B.; Tinôco, I. de F. F.; Menegali, I.; Souza J. J.; Holanda, M. Avaliação do uso de ventilação mínima em galpões avícolas e de sua influência no desempenho de aves de corte no período de inverno. Revista Brasileira de Zootecnia, v.39, p.13811386. 2010. http://dx.doi.org/10.1590/S1516-35982010000600030

Wathes, C. M. Strive for clean air in your poultry house. World Poultry, v.15, p.17-19. 1999.

Wathes, C. M.; Phillips, V. R.; Holden, M. R.; Sneath, R. W.; Short, J. L.; White, R. P. P. Emissions of aerial pollutants in livestock buildings in Northern Europe: Overview of a multinational project. Journal of Agricultural Engineering Research, v.70, p.3-9. 1998. http://dx.doi. org/10.1006/jaer.1998.0278 
WHO - Word Health Organization. Guías de calidad del aire de la OMS relativas al material particulado, el ozono, el dióxido de nitrógeno y el dióxido de azufre: actualización mundial. 2005. <http://apps.who.int/iris/handle/10665/69478>. 15 Jan. 2015.
Zapata, O. L.; Tinôco, I. de F. F.; Osorio, J. A.; Souza, C. F.; Vieira, M. F. Evaluation of the fertilizer and contamination potential of different broiler litter types subjected to various use cycles. Revista Facultad Nacional de Agronomia, v.68, p.7637-7646. 2015. http:// dx.doi.org/10.15446/rfnam.v68n2.50967 